Portland State University

PDXScholar

$5-1-2002$

\title{
The Meyer-Neldel rule for a property determined by two transport mechanisms
}

\author{
Ralf Widenhorn \\ Portland State University \\ Armin Rest \\ Erik Bodegom \\ Portland State University
}

Follow this and additional works at: https://pdxscholar.library.pdx.edu/phy_fac

Part of the Physics Commons

Let us know how access to this document benefits you.

\section{Citation Details}

Widenhorn, R., Rest, A., \& Bodegom, E. (2002). The Meyer-Neldel rule for a property determined by two transport mechanisms. Journal Of Applied Physics, 91(10), 6524.

This Article is brought to you for free and open access. It has been accepted for inclusion in Physics Faculty Publications and Presentations by an authorized administrator of PDXScholar. Please contact us if we can make this document more accessible: pdxscholar@pdx.edu. 


\title{
The Meyer-Neldel rule for a property determined by two transport mechanisms
}

\author{
Ralf Widenhorn ${ }^{\text {a) }}$ \\ Department of Physics, Portland State University, Portland, Oregon 97207-0751 \\ Armin Rest \\ Department of Astronomy, University of Washington, Seattle, Washington 98195 \\ Erik Bodegom ${ }^{\text {b) }}$ \\ Department of Physics, Portland State University, Portland, Oregon 97207-0751
}

(Received 27 December 2001; accepted for publication 22 February 2002)

\begin{abstract}
We propose that the Meyer-Neldel rule (MNR) arises naturally for a quantity where both an intrinsic process as well as a process involving impurities contribute. The strength of the latter depends solely on the density of the impurities. This leads to a spread in the apparent activation energy of the measured quantity and the observation of the MNR, even though the intrinsic processes have fixed activation energies. A consequence of the MNR is the occurrence of a temperature $T_{\mathrm{MN}}$ where a measured parameter is independent of the activation energy. For the system studied, the MNR does not accurately predict the results at temperatures larger than $T_{\mathrm{MN}}$. Our model for the MNR is supported by experimental data and it also can explain the inverse MNR for low activation energies. (C) 2002 American Institute of Physics. [DOI: 10.1063/1.1469666]
\end{abstract}

\section{INTRODUCTION}

Various physical processes are thermally activated and therefore strongly temperature dependent. It is often observed that an exponential power law in the form

$$
X=X_{0} \exp (-\Delta E / k T)
$$

can describe the temperature dependence of a physical property, $X$. The activation energy $\Delta E$ is generally determined by a barrier height or the band gap between two states. Defects or doping in, for example, semiconductors, can lead to lower effective activation energies and to a spread in the values for $\Delta E$ for the same property in one material. Meyer and Neldel observed in 1937 a relation between the exponential prefactor $X_{0}$ and $\Delta E$ in related processes: ${ }^{1}$

$$
X_{0}=X_{00} \exp \left(\Delta E / E_{\mathrm{MN}}\right),
$$

where $X_{00}$ and $E_{\mathrm{MN}}$ are positive constants. Since then, the origin of this relation [referred to as the Meyer-Neldel rule (MNR)] and the physical interpretations of $X_{00}$ and $E_{\mathrm{MN}}$ have been the subject of speculations. Processes where $E_{\mathrm{MN}}<0$ are rarer and referred to as inverse or anti-MeyerNeldel rule. ${ }^{2-6}$

The discussion of whether there is one universal explanation for the MNR in different systems is not yet settled. Various plausible models have been proposed. One common interpretation is based on an exponential density of states distribution that induces a shift in the Fermi level. ${ }^{7}$ While this approach is appealing for some experiments, the MNR is more widespread than predicted by this model. For example, Fortner et al. ${ }^{8,9}$ showed that it cannot explain the MNR for liquid semiconductors. Also, for various experiments, this

\footnotetext{
${ }^{a}$ Electronic mail: ralfw@pdx.edu

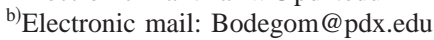

model results in physically unreasonable values for $X_{0} \cdot{ }^{10}$ Others speculate that the MNR arises because of the entropy of multiple excitations. ${ }^{11-13}$ Though this approach is very general, it cannot be an universal explanation for the MNR. ${ }^{14}$ Substituting Eq. (2) into Eq. (1) leads to $X$ $=X_{00} \exp \left(\left[1 / E_{\mathrm{MN}}-1 / k T\right] \Delta E\right)$. One sees immediately that for a characteristic temperature $T_{\mathrm{MN}}=E_{\mathrm{MN}} / k$, the process becomes independent of the activation energy. In a plot of $\ln (X)$ versus the inverse temperature (Arrhenius plot), the lines of different samples should show one common intersection at $T_{\mathrm{MN}}$. At this temperature, $X$ is independent of the activation energy. Most experiments have reported results measured at temperatures lower than $T_{\mathrm{MN}}$, i.e., the values for $X$ merge as the temperature increases. Some experiments on liquid semiconducting alloys exhibited values where the measured temperature range is higher than $T_{\mathrm{MN}}{ }^{8,9,14}$ To our knowledge, nobody has convincing data of a process where temperatures both below and above $T_{\mathrm{MN}}$ yielded the same value for the characteristic temperature $T_{\mathrm{MN}}$. It is therefore unknown if the intersection at $T_{\mathrm{MN}}$ can be observed. Some factors make it difficult to measure this intersection: Since $X$ generally varies by several orders of magnitude, it is usually very difficult to measure its value over a wide temperature range. Furthermore, material characteristics might change drastically with temperature because phase transitions like melting or evaporation can occur. Nonetheless, we believe that if such an intersection is possible one should be able to observe it for some systems.

Frequently, it is observed that the Arrhenius plot deviates from a linear behavior in the form of a positive curvature. ${ }^{15-23}$ Either experimental uncertainties, sparse data, a narrow temperature range, or a combination of these issues often make it impossible to determine a distinct curvature. The origin of the nonlinearity may vary from experiment to 
experiment. Yoon and Lee ${ }^{24}$ argued that the statistical shift of the Fermi level can cause the bending. Another common explanation for the bending is that the activation path changes with temperature. ${ }^{25}$ Some found a linear dependence of $\ln (X)$ with $T^{-1 / 4}$ at low temperatures and identified the transport mechanism as dominated by variable range hopping, which is described by Mott's law. ${ }^{15,17,18}$ It has also been shown that an excitation involving multiple acceptor levels ${ }^{26}$ or midgap impurities ${ }^{27}$ results in a temperature-dependent activation energy. At one particular temperature, one excitation might dominate, however, one or more other paths contribute to $X$ as well.

It is frequently found that the value for $E_{\mathrm{MN}}$ correlates with the temperature covered by the particular experiment. ${ }^{28,29}$ It has been shown that approximations in the Arrhenius plot can result in a MNR behavior with $E_{\mathrm{MN}}$ $=k T^{*}$, where $T^{*}$ is equal to a particular temperature within the experimental temperature range. ${ }^{28,30}$ It has also been shown that the MNR of diffusion experiments are due to experimental error. ${ }^{31}$

In our opinion, it is not necessary that there is one universal explanation of the MNR. In order to evaluate what causes it in each single published experiment, the data has to be reported in great detail. Only if the Arrhenius plots with error bars are presented can the possibility of experimental error be excluded. We will present a model for the MNR, which is consistent with experimental data that exhibit a slight curvature in the Arrhenius plot and no actual intersection at $T_{\mathrm{MN}}$. The change of the main activation path with temperature, due to multiple processes coexisting at the same time, leads to the MNR. In particular, we investigate the case of an excitation involving a monoenergetic impurity distribution at the mid gap of silicon. Based on parameters obtained experimentally for the dark current in a charge-coupled device (CCD) chip, ${ }^{27}$ we will generate Arrhenius-type plots for different impurity concentrations. The computed data are fitted with a least square fitting routine according to the Arrhenius law [Eq. (2)]. We will also derive an analytic approximation to the model data in the form of a first order Taylor series. Based on the Taylor series, an analytic expression for $E_{\mathrm{MN}}$ is derived and found to be $k T$, where $T$ is in the order of the temperature range covered by the experiment. Finally, we will show that a linear fit of $\ln (X)$ versus the inverse temperature to the model data, the Taylor series, as well as the experimental data, lead to the same MNR.

\section{MODEL}

We assume that there is a measured property $X$ for a group of related samples which have one transport mechanism in common:

$$
X_{a}=X_{a 0} \exp \left(-\Delta E_{a} / k T\right) .
$$

Another transport path, coexisting in the material, contributes to $X$ as well. This transport mechanism depends on the conditions at which the particular sample was treated. For example, the amount of annealing, doping, or the diffusion of impurities can determine its magnitude. Its value varies for different samples of a material. In an experiment, one can only measure the property $X$, as it is given by the sum of both mechanisms. The variation in the second transport mechanism will cause a spread in the effective activation energies, which is determined by a straight line fit of $\ln (X)$ versus the inverse temperature. We will investigate in greater detail the case where the contribution of the second transport mechanism can be expressed by a function which is in form identical to Eq. (3):

$$
X_{b}=X_{b 0} \exp \left(-\Delta E_{b} / k T\right) .
$$

For now we will assume a general temperature dependent function $X_{b}$, which represents the second mechanism. The total value for $X$ is given by the sum of $X_{a}$ and $X_{b}$. In the Arrhenius plot, the logarithm of $X$ is plotted versus the inverse temperature. Therefore, we define $f \equiv \ln (X)$ and $f^{\prime}$ $\equiv \partial f / \partial(1 / k T)$. By fitting the logarithm of $X$ as a function of the inverse temperature with a straight line, one calculates a linear approximation of $f$ for the measured temperature range. Analytically, one can calculate a linear approximation of $f$ by a first order Taylor series expansion around a specific temperature $T_{m}$ (or inverse energy $1 / k T_{m}$ ). The Taylor series of $f$ is given by

$$
\begin{aligned}
f(T)= & f\left(T_{m}\right)+f^{\prime}\left(T_{m}\right)\left(\frac{1}{k T}-\frac{1}{k T_{m}}\right) \\
& +\frac{1}{2} f^{\prime \prime}\left(T_{m}\right)\left(\frac{1}{k T}-\frac{1}{k T_{m}}\right)^{2}+\cdots .
\end{aligned}
$$

Naming the first order Taylor expansion $g$ and regrouping the terms, we get

$$
\begin{aligned}
g & =f^{\prime}\left(T_{m}\right) / k T+\left[f\left(T_{m}\right)-f^{\prime}\left(T_{m}\right) / k T_{m}\right] \\
& =-\Delta E_{\text {Tay }} / k T+\ln \left(X_{0, \text { Tay }}\right) .
\end{aligned}
$$

For a good linear approximation of $f, T_{m}$ should be approximately in the middle of the investigated temperature range. $\Delta E_{\text {Tay }}$ is the apparent activation energy which is given by

$$
\begin{aligned}
& \Delta E_{\text {Tay }}=-f^{\prime}\left(T_{m}\right): \\
& X_{0, \text { Tay }}=\exp \left[f\left(T_{m}\right)-f^{\prime}\left(T_{m}\right) / k T_{m}\right]
\end{aligned}
$$

is the exponential prefactor as given in Eq. (1). Combining Eqs. (6) and (7), it follows:

$$
\ln \left(X_{0, \text { Tay }}\right)=\ln \left[X\left(T_{m}\right)\right]+\Delta E_{\text {Tay }} / k T_{m} .
$$

The MNR results if $\ln \left(X_{0, \text { Tay }}\right)$ is linearly dependent on $\Delta E_{\text {Tay }}$ with $E_{\mathrm{MN}}$ as the constant of proportionality. Note that $\ln \left[X\left(T_{m}\right)\right]$ in Eq. (8) generally contains an implicit dependence on $\Delta E_{\text {Tay }}$ which we will discuss in Sec. III.

\section{RESULTS AND DISCUSSION}

We assume that two independent transport mechanisms, obeying Eqs. (3) and (4), contribute to the property $X$. One mechanism is determined by an intrinsic activation and the other by an activation that involves impurities:

$$
X=X_{a 0} \exp \left(-\Delta E_{a} / k T\right)+X_{b 0} \exp \left(-\Delta E_{b} / k T\right) .
$$

We assume further that the activation energy for the intrinsic activation $\Delta E_{a}$ and the exponential prefactor $X_{a 0}$ are constant. The energy level of the impurities $\Delta E_{b}$ is set constant 


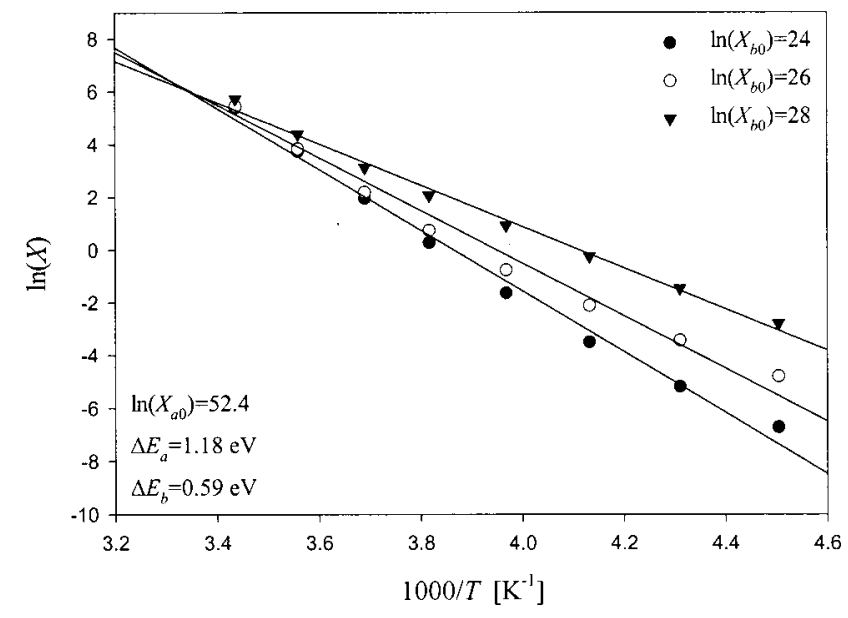

FIG. 1. $\ln (X)$ vs the inverse temperature for three different $\ln \left(X_{b 0}\right) \mathrm{s}$ and linear fits to the data points.

$\left(\Delta E_{b}<\Delta E_{a}\right)$ as well. By varying the number of impurities (actually we vary $X_{b 0}$ ) we can obtain Arrhenius-type plots and determine the conditions under which one observes the MNR. Since we want to compare the model data to a real experiment, we chose parameters which are realistic for dark current in a CCD imager. ${ }^{27}$ The dark current $X$ and the prefactors $X_{a 0}$ and $X_{b 0}$ are given in counts/s. Since the units of $X, X_{a 0}$, and $X_{b 0}$ are not important to our analysis, we will consider their values dimensionless. A fit based on two independent thermally activated transport mechanisms to the dark current in a CCD chip between 222 and $291 \mathrm{~K}$ resulted in two major distributions of activation energies. For the computer simulation we fixed three parameters at the peak of these distributions: $\Delta E_{a}=1.18 \mathrm{eV}, \ln \left(X_{a 0}\right)=52.4$, and $\Delta E_{b}$ at midgap $(0.59 \mathrm{eV})$. All parameters are realistic for a CCD chip. Midgap impurity levels have been found in other CCDs and can, for example, be caused by $\mathrm{Au}, \mathrm{Ni}$, or $\mathrm{Co}$ contamination. ${ }^{32,33}$

The data points in Fig. 1 are generated with Eq. (9). In an actual experiment one does not know about the two mechanisms and a linear fit according to Arrhenius law [Eq. (1)] seems reasonable. Inevitable uncertainties in the actual measurement would also make it more difficult to detect the deviation from Eq. (1). The lines for impurity densities at the depicted impurity concentrations appear to merge with increasing temperatures. We will show that a linear fit to these lines will lead to a common apparent intersection at $T$ $=T_{\mathrm{MN}}$. The observation of the MNR requires, therefore, only the assumption of different impurity concentrations.

With a least square fit, according to Eq. (1), to the model data generated by Eq. (9), one can find values for the prefactor $X_{0 \text { fit }}$ and the apparent activation energy $\Delta E_{\text {fit }}$. Figure 2 shows that the relation between $\ln \left(X_{0 \mathrm{fit}}\right)$ and the apparent activation energy $\Delta E_{\text {fit }}$ while almost reaching from the impurity level $\Delta E_{b}$ to the intrinsic activation energy $\Delta E_{a}$ can be described linearly over a very wide range (see closed circles). The minimum in $\Delta E_{\text {fit }}$ is reached for $\ln \left(X_{b 0}\right)$ $=29.6$. For higher impurity concentrations one can observe the inverse MNR with a slope approaching negative infinity. A similar inverse MNR was found by Meiling and Schropp, ${ }^{3}$

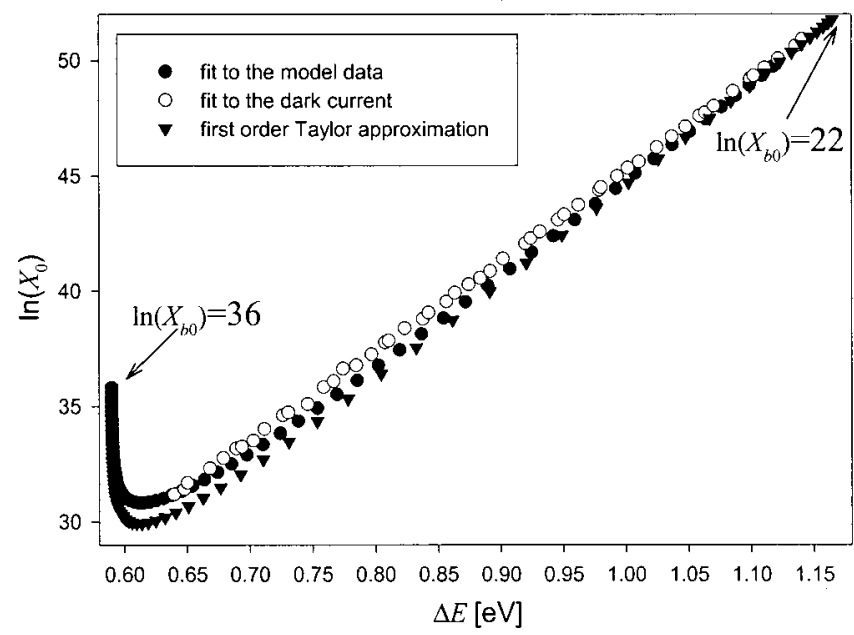

FIG. 2. $\ln \left(X_{0}\right)$ vs $\Delta E$ for the dark current in a CCD and for the model data based on $\ln \left(X_{b 0}\right) \mathrm{s}$ in 0.2 increments between 22 and 36 .

for thin-film transistors with intrinsic heterogeneous silicon.

As mentioned earlier, the exponential approximation [Eq. (1)] can also be obtained from a first order Taylor series [Eq. (5)]. For Eq. (9), the apparent activation energy $\Delta E_{\text {Tay }}$ [Eq. (6)] is given by

$\Delta E_{\text {Tay }}$

$$
=\frac{\Delta E_{a} X_{a 0} \exp \left(-\Delta E_{a} / k T_{m}\right)+\Delta E_{b} X_{b 0} \exp \left(-\Delta E_{b} / k T_{m}\right)}{X_{a 0} \exp \left(-\Delta E_{a} / k T_{m}\right)+X_{b 0} \exp \left(-\Delta E_{b} / k T_{m}\right)} .
$$

Considering all other parameters as fixed, $\Delta E_{\text {Tay }}$ is monotonous in $X_{b 0} . \Delta E_{\text {Tay }}$ decreases from $\Delta E_{a}$ for small values of $X_{b 0}$ [see solid triangles in Fig. 2 with $\left.\ln \left(X_{b 0}\right)=22\right]$ to $\Delta E_{b}$ for high impurity concentrations [see solid triangles in Fig. 2 with $\left.\ln \left(X_{b 0}\right)=36\right]$. The Taylor series is expanded around $T_{m}$ $=259 \mathrm{~K}$, hence approximately in the middle of the used temperature range. We found that at $T_{m}$ the least square fit (solid circles in Fig. 2) and the Taylor series (solid triangles in Fig. 2) have similar activation energies and prefactors for the same $X_{b 0}$ s. It is remarkable that the MNR, as shown by both data series, is the result of varying impurity concentrations and a reasonable linear approximation only. Figure 2 also shows that the model data analyzed with the Taylor series and the fit are a good approximation to the actual results obtained by dark current measurements in a CCD. The figure depicts 52 arbitrary data points (out of 222784 data points for the $492 \times 492$ pixel subframe), chosen such that a wide range of activation energies is covered. The characteristic Meyer-Neldel energy $E_{\mathrm{MN}}$, as the slope of the linear region in Fig. 2, is very close to the thermal energy at the temperature of the data sets. Looking only at the least square fits, this correspondence is not obvious, but the reason for this can be found in the Taylor series analysis. The Meyer-Neldel energy can be calculated as the slope of $\ln \left(X_{0, \text { Tay }}\right)$ vs $\Delta E_{\text {Tay }}$. Using Eq. (8) it follows: 


$$
\begin{aligned}
\frac{d \ln \left(X_{0, \text { Tay }}\right)}{d \Delta E_{\text {Tay }}} & =\frac{d \ln \left(X_{0, \text { Tay }}\right)}{d X_{b 0}}\left(\frac{d \Delta E_{\text {Tay }}}{d X_{b 0}}\right)^{-1} \\
& =\left[\frac{d \ln \left[X\left(T_{m}\right)\right]}{d X_{b 0}}+\frac{1}{k T_{m}} \frac{d \Delta E_{\text {Tay }}}{d X_{b 0}}\right]\left(\frac{d \Delta E_{\text {Tay }}}{d X_{b 0}}\right)^{-1} .
\end{aligned}
$$

The derivative of Eq. (10) with respect to $X_{b 0}$ is given by

$$
\begin{aligned}
& \left(\frac{d \Delta E_{\text {Tay }}}{d X_{b 0}}\right) \\
& \quad=\frac{X_{a 0} \exp \left(-\Delta E_{a} / k T_{m}\right) \exp \left(-\Delta E_{b} / k T_{m}\right)\left(\Delta E_{b}-\Delta E_{a}\right)}{\left[X_{a 0} \exp \left(-\Delta E_{a} / k T_{m}\right)+X_{b 0} \exp \left(-\Delta E_{b} / k T_{m}\right)\right]^{2}}
\end{aligned}
$$

and

$$
\begin{aligned}
& \frac{d \ln \left[X\left(T_{m}\right)\right]}{d X_{b 0}} \\
& \quad=\frac{\exp \left(\Delta E_{b} / k T_{m}\right)}{X_{a 0} \exp \left(-\Delta E_{a} / k T_{m}\right)+X_{b 0} \exp \left(-\Delta E_{b} / k T_{m}\right)} .
\end{aligned}
$$

Combining Eqs. (11), (12) and (13), it follows:

$$
\begin{aligned}
E_{\mathrm{MN} / \mathrm{Tay}}^{-1}= & 1 / k T_{m}+\left(\Delta E_{b}-\Delta E_{a}\right)^{-1} \\
& \times\left[1+X_{b}\left(T_{m}\right) / X_{a}\left(T_{m}\right)\right] .
\end{aligned}
$$

The second term in Eq. (14) is monotonous in $X_{b 0}$, negative, and over a wide range of $X_{b 0} \mathrm{~s}$ and $\Delta E_{\mathrm{Tay}} \mathrm{s}$ small compared to $1 / k T_{m} . E_{\mathrm{MN}}$ is therefore slightly higher than $k T_{m}$ for all activation energies. As also seen in Fig. 2, $E_{\mathrm{MN} \text {,Tay }}$ calculated with Eq. (14) is close to the experimental value for $E_{\mathrm{MN}}$ of $25.3 \mathrm{meV}$ (given as the inverse of the slope of all 222784 data points in a graph similar to Fig. 2). Generally one can observe a MNR when the following applies in the measured temperature range: $X_{b}$ and $X_{a}$ are such that there is a transition from the intrinsic to the secondary process for the given impurity concentrations, the Arrhenius plots are reasonably linear, and $E_{\mathrm{MN}}$ is determined by the first term in Eq. (14).

The model data also explain that the dark current measurements at a temperature $(k T=27 \mathrm{meV})$ higher than the calculated critical temperature $\left(k T_{\mathrm{MN}}=25.3 \mathrm{meV}\right)$ did not show a crossover in the Arrhenius plot as predicted by the MNR. ${ }^{27}$ Considering the MNR only, one would expect for $T>T_{\mathrm{MN}}$ an increasing value for $X$ with increasing activation energy. However, we could only observe that $\ln (X)$ was converging for all pixels. This means for $T>T_{\mathrm{MN}}$ the intrinsic transport is dominant and the different impurity concentrations do not contribute strongly to the value of $\ln (X)$.

Our model is not limited to the system described above. For example, multiple impurity levels with different energies, which depend on the kind of impurities, and different prefactors, which depend on their prevalence, could contribute to the total count. Thus, $X=\sum_{i=0}^{n} X_{i 0} \exp \left(-\Delta E_{i} / k T\right)$ would show the same characteristics in a limited temperature range as two exponential functions only: converging values with increasing temperatures, a slight positive curvature in the Arrhenius plot, and the observation of the MNR. We also found that other functions for $X_{b}$ [Eq. (4)] lead to the same result. For example, $X_{b}=X_{b 0} \exp \left[-\left(T_{h} / T\right)^{1 / 4}\right]$, for variable range hopping, as described by Mott's law, results for different $X_{b 0}$ s in a MNR plot similar to Fig. 2.

Experiments, where the measured temperatures are larger than the characteristic temperature $T_{\mathrm{MN}}{ }^{8,9,14}$ can be explained by a change in the values of $X_{a 0}$. Approximately constant values of $X_{b 0}$ and a varying $X_{a 0}$ cause a merging of $X$ at lower temperatures. The apparent intersection at $T_{\mathrm{MN}}$ is thus lower than the measured temperatures.

\section{CONCLUSIONS}

We showed that a physical property which is the sum of two activation mechanisms, a mediated mechanism and an intrinsic mechanism, can cause a spread in the effective activation energy. Variations in the impurity concentration (i.e., different prefactors for the mediated process) result in effective activation energies and effective prefactors that showed MNR behavior and inverse MNR behavior. Thus, despite the fact that the underlying activation energies do not obey the MNR, the MNR is observed in the apparent activation energies. The characteristic Meyer-Neldel energy $E_{\mathrm{MN}}$ was calculated as approximately $k T$, with $T$ in the investigated temperature range. Our model quite accurately mimicked the experimentally observed MNR. The assumption of two or multiple independent processes contributing to a physical property is expected to be generally applicable. We expect, therefore, that the model could be an explanation of the observation of the MNR and the inverse MNR in other systems as well.

${ }^{1}$ W. Meyer and H. Neldel, Z. Tech. Phys. (Leipzig) 12, 588 (1937).

${ }^{2}$ R. Flückiger, J. Meier, M. Goetz, and A. Shah, J. Appl. Phys. 77, 712 (1995).

${ }^{3}$ H. Meiling and R. E. I. Schropp, Appl. Phys. Lett. 74, 1012 (1999).

${ }^{4}$ J. H. Schön, Ch. Kloc, and B. Batlogg, Phys. Status Solidi B 121, R4 (2000).

${ }^{5}$ G. Lucovsky, C. Wang, M. J. Williams, Y. L. Chen, and D. M. Maher, in Microcrystalline Semiconductor; Materials Sciences and Devices, edited by F. M. Faucket, C. C. Tsai, L. T. Canham, I. Shimuzu, and Y. Auyag, Mater. Res. Soc. Symp. Proc. No. 283 (Materials Research Society, Pittsburgh, 1993).

${ }^{6}$ A. Rubino, M. L. Addonizio, G. Conte, G. Nobile, E. Terzini, and Madan, in Amorphous Silicon Technology 1993, edited by E. A. Schiff, M. J. Thompson, A. Madding, K. Tanaka, and Agnala Le Comber, Mater. Res. Soc. Symp. Proc. No. 297 (Materials Research Society, Pittsburgh, 1993).

${ }^{7}$ H. Overhof and P. Thomas, Electronic Transport in Hydrogenated Amorphous Semiconductors (Springer, Berlin, 1989).

${ }^{8}$ J. Fortner, M.-L. Saboungi, and J. E. Enderby, Philos. Mag. Lett. 68, 85 (1993).

${ }^{9}$ J. Fortner, V. G. Karpov, and M.-L. Saboungi, Appl. Phys. Lett. 66, 997 (1995).

${ }^{10}$ A. Yelon, B. Movaghar, and H. M. Branz, Phys. Rev. B 46, 12244 (1992).

${ }^{11}$ A. Yelon and B. Movaghar, Phys. Rev. Lett. 65, 618 (1990).

${ }^{12}$ G. Boisvert, L. J. Lewis, and A. Yelon, Phys. Rev. Lett. 75, 469 (1995).

${ }^{13}$ A. Yelon and B. Movaghar, Appl. Phys. Lett. 71, 3549 (1997).

${ }^{14}$ D. You, H. S. Schnyders, and J. B. Zytveld, J. Phys.: Condens. Matter 9, 1407 (1997).

${ }^{15}$ D. H. Tassis, C. A. Dimitriadis, and O. Valassiades, J. Appl. Phys. 84, 2960 (1998)

${ }^{16}$ K. Morii, T. Matsui, H. Tsuda, and H. Mabuchi, Appl. Phys. Lett. 77, 2361 (2000).

${ }^{17}$ C. Guillén and J. Herrero, J. Appl. Phys. 71, 5479 (1992).

${ }^{18}$ D. H. Tassis, C. A. Dimitriadis, J. Brini, G. Kamarinos, and A. Birbas, J. Appl. Phys. 85, 4091 (1999).

${ }^{19}$ D. G. Papageorgiou and G. A. Evangelakis, Surf. Sci. Lett. 461, 543 (2000). 
${ }^{20}$ E. Quartarone, P. Mustarelli, C. Tomasi, and A. Magistris, J. Phys. Chem. B 102, 9610 (1998).

${ }^{21}$ J. C. Wang and Y. F. Chen, Appl. Phys. Lett. 73, 948 (1998).

${ }^{22}$ E. J. Meijer, M. Matters, P. T. Herwig, D. M. de Leeuw, and T. M. Klapwijk, Appl. Phys. Lett. 76, 3433 (2000).

${ }^{23}$ P. A. W. E. Verleg and J. I. Dijkhuis, Phys. Rev. B 58, 3917 (1998).

${ }^{24}$ B.-G. Yoon and C. Lee, J. Appl. Phys. 60, 673 (1986).

${ }^{25}$ Y. Lubianiker and I. Balberg, Phys. Rev. Lett. 78, 2433 (1997).

${ }^{26}$ K. Herz and M. Powalla, Appl. Surf. Sci. 91, 87 (1995).

${ }^{27}$ R. Widenhorn, L. Mündermann, A. Rest, and E. Bodegom, J. Appl. Phys. 89, 8179 (2001).

${ }^{28}$ R. Kirchheim and X. Y. Huang, Phys. Status Solidi B 144, 253 (1987).
${ }^{29}$ X. L. Wu, R. Shinar, and J. Shinar, Phys. Rev. B 44, 6161 (1991).

${ }^{30}$ P. Mialhe, J. P. Charles, and A. Khoury, J. Phys. D 21, 383 (1988).

${ }^{31}$ D. J. Dunstan, Solid State Commun. 107, 159 (1998).

${ }^{32}$ W. C. McColgin, J. P. Lavine, and C. V. Stancampiano, in DefectImpurity-Engineered Semiconductors and Devices, edited by S. Ashok, J. Chevallier, I. Akasaki, N. M. Johnson, and B. L. Sopori, Mater. Res. Soc. Symp. Proc. No. 378 (Materials Research Society, Pittsburgh, 1995).

${ }^{33}$ W. C. McColgin, J. P. Lavine, C. V. Stancampiano, and J. B. Russell, in Defect Impurity Semiconductors and Devices II, edited by S. Ashok, J. Chevallier, K. Sumino, B. L. Sopori, and W. Goetz, Mater. Res. Soc. Symp. Proc. No. 510 (Materials Research Society, Pittsburgh, 1998). 